\title{
Fast BEM Analysis of Porous Solids
}

\author{
Zaiyou Yan ${ }^{\mathrm{a}, \mathrm{b}}$, Jun Zhang ${ }^{\mathrm{a}}$, Wenjing Ye ${ }^{\mathrm{a},}$ and T.X. Yu ${ }^{\mathrm{a}}$ \\ ${ }^{a}$ Department of Mechanical Engineering, Hong Kong University of Science and Technology \\ *mewye@ust.hk \\ ${ }^{b}$ Department of Aerodynamics, Nanjing University of Aeronautics \& Astronautics
}

\begin{abstract}
A fast boundary integral approach based on the precorrect FFT technique is developed for the 3-D linear elastic analysis of porous solids. Compared to the conventional boundary element method, this approach is much more efficient as both the computational time and memory usage are greatly reduced. Preliminary study on the Young's modulus and Poisson ratio of porous solids with spherical cavities is conducted. Effects of cavity size and distribution on these properties are investigated using the developed BEM code.
\end{abstract}

Keywords: Boundary element method, pre-corrected FFT technique, porous solids.

PACS: 45.10. -b; , 81.05.Rm

\section{INTRODUCTION}

Mechanical characterization of porous solids is an essential step in the applications of such an important type of materials. Owing to the random distribution of the pores and their irregular shapes and varied sizes, the analytical approaches often rely on simplified models and the assumption of the existence of a representative cell. While these elegant approaches reveal insightful relationships between some key parameters, the quantitative prediction of the mechanical properties may not be accurate enough in some applications. With the advent of advanced numerical techniques and rapidly developed computing power, detailed three-dimension numerical modeling of realistic porous solids has become increasingly popular due to its capability to produce accurate predictions of mechanical responses of these structures and its capability of capturing the effects due to non-uniform distribution, irregular shapes and size on the overall behavior of porous solids. Such a trend can be clearly observed from the literature where the recent modeling effort has been shifted from analytical analysis towards numerical simulations [1,2].

It is perhaps fair to say that the current leading method in the simulation of porous solids is the finite element method (FEM). Indeed, FEM is a mature, powerful and versatile method suitable for an extremely wide scope of problems. However, one challenge in using the FEM is the generation of good-quality volume fitting mesh for problems with complex 3-D domains. With irregular shapes and random distribution of pores, porous solids, particularly those with large porosity, could be one of these examples in which a good quality volume mesh is difficult to produce. The boundary element method (BEM) [3], on the other hand, requires only surface mesh for linear problems. It is thus particularly suitable for porous solids because the surface mesh for each pore and the solid phase can be generated independently and parallel, greatly reducing the mesh complexity. In addition, the recent developed accelerated techniques, such as the fast multipole method [4], the precorrected Fast Fourier Transformation (PFFT) technique [5], when combined with iterative linear system solvers, have greatly reduced the computational time and the memory usage required in solving the final discretized system, making large-scale problems tacklable using the BEM. Successful applications of the accelerated BEM in solving large scale problems can be found in the areas of Microelectromechanical systems [6], composite materials [7], etc.

This paper reports our work on the development of a PFFT-accelerated BEM approach for 3-D linear elastic analysis of porous solids. The objective is to develop an efficient numerical method that allows a systematic numerical study of the distribution, size and irregular shapes of pores on the overall mechanical behavior of porous structures. 


\title{
NUMERICAL METHODS AND IMPLEMENTATION
}

\author{
BEM Model for Elastostatics
}

The conventional direct boundary element formulation [3] for 3-D linear elastostatic problems is

$$
c_{i j}(p) u_{j}(p)=\iint_{S}\left[G_{i j}(p, q) \tau_{j}(q)-F_{i j}(p, q) u_{j}(q)\right] d S_{q}
$$

with

$$
c_{i j}(p)=-\iint_{S} F_{i j}(p, q) d S_{q} .
$$

The integral kernels $G_{i j}$ and $F_{i j}$ in the formulation are

$$
\begin{gathered}
G_{i j}(p, q)=\frac{C_{1}}{r(p, q)}\left(C_{3} \delta_{i j}+\frac{\partial r(p, q)}{\partial x_{i}} \frac{\partial r(p, q)}{\partial x_{j}}\right) \\
F_{i j}(p, q)=-\frac{C_{2}}{r^{2}(p, q)}\left\{\frac{\partial r(p, q)}{\partial n_{q}}\left(C_{4} \delta_{i j}+3 \frac{\partial r(p, q)}{\partial x_{i}} \frac{\partial r(p, q)}{\partial x_{j}}\right)+C_{4}\left(n_{i} \frac{\partial r(p, q)}{\partial x_{j}}-n_{j} \frac{\partial r(p, q)}{\partial x_{i}}\right)\right\},
\end{gathered}
$$

where $u_{j}$ and $\tau_{j}$ represent the $j$-th component of the displacement and the traction, $p$ and $q$ are the source point and field point and $r=|p-q|$ is the distance between these two points. The four constants are given as

$$
C_{1}=\frac{1}{16 \pi \mu(1-v)}, C_{2}=\frac{1}{8 \pi(1-v)}, C_{3}=3-4 v, C_{4}=1-2 v,
$$

where $\mu=\frac{E}{2(1+v)}, E$ and $v$ are the Young's modulus and the Poisson ratio respectively. For points on smooth surface, $c_{i j}(p)=\frac{1}{2} \delta_{i j}$.

\section{The Precorrected FFT Technique}

In the precorrected FFT (PFFT) technique, a parallelepiped is constructed to enclose a three-dimensional problem after it has been discretized into $n$ surface panels. This parallelepiped is then subdivided into an $k \times l \times m$ array of small cubes so that each small cube contains only a few panels. It should be noted that the surface panels and the PFFT cubes can intersect with each other and there is no need to maintain any consistency between the surface panels and the cubes.

The acceleration of surface integration is achieved by exploiting the fact that the kernels in the surface integrals such as those in Equation (1) have piecewise-smooth convolutional form. Thus with the aid of the uniform grid formed by the cubes in the parallelepiped, these integrals can be computed approximately using the Fast Fourier Transform technique. To ensure accuracy, such an approximation is only employed for far-field interactions (i.e., integrals in which the source point is far away from the field panel). For nearby interactions, direct evaluation is required. For a detailed description of this technique, readers are referred to [5].

\section{Numerical Implementation}

The randomly distributed pores are generated using the random sequential adsorption (RSA) algorithm [8]. This algorithm generates one pore at a time and the distance between this pore and all the others generated previously must be larger than a pre-set value. In addition, a minimum distance between pores and solid boundaries can also be set. If a unit cell with a periodic boundary condition is considered, extra care must be taken to ensure that these conditions are met. The RSA algorithm is very easy to implement and generates pretty isotropic distribution. However, there is an inherent limit on the largest porosity that can be generated using this algorithm. For identical pores, the maximum porosity is around $52 \%$.

Equation (1) is solved using a boundary element method together with the PFFT acceleration technique. For simplicity, a piece-wise constant collocation scheme is used to discretize the integral equation. The surface of the 
structure is discretized into small panels. On each panel, $\mathbf{u}$ and $\tau$ are assumed to be constant. A system of equations for the panel unknowns is then derived by insisting that the integral equations are satisfied at each panel centroid. The resultant linear system is then solved using the generalized minimal residual method (GMRES) [9] combined with the PFFT technique. Weakly singular integrals in kernel $G_{i j}$ are treated using the classical polar coordinate transformation method and the strongly singular integrals in kernel $F_{i j}$ are computed using the analytical formulation presented by Cruse [10].

\section{Numerical Results}

\section{Validation of the BEM Code}

Case1: First we validate the in-house accelerated BEM code using a classical example: a pressurized thick-walled cylinder [12] as shown in Fig. 1. The inner and the outer radii of the cylinder are $10 \mu \mathrm{m}$ and $25 \mu \mathrm{m}$ respectively.

The height is $10 \mu \mathrm{m}$. The elastic modulus of the material is $1.65 \times 10^{5} \mathrm{MPa}$ and the Poisson ratio is 0.33 . In this case, the inner surface of the cylinder is subject to a $1 \mathrm{MPa}$ pressure while the outer surface is traction free. The analytical solutions for this case are

$$
\sigma_{r}=\frac{1}{r_{2}^{2}-r_{1}^{2}}\left[p_{1} r_{1}^{2}-\frac{r_{1}^{2} r_{2}^{2} p_{1}}{r^{2}}\right], \sigma_{\theta}=\frac{1}{r_{2}^{2}-r_{1}^{2}}\left[p_{1} r_{1}^{2}+\frac{r_{1}^{2} r_{2}^{2} p_{1}}{r^{2}}\right], u_{r}=\frac{r}{E}\left(\sigma_{\theta}-v \sigma_{r}\right)
$$

The accuracy and the convergence of the accelerated BEM are illustrated in Table 1 where the mean values of the radial displacements on the two surfaces $r=10 \mu \mathrm{m}$ and $r=25 \mu \mathrm{m}$, obtained from various methods, are presented. It is clear that the accuracy of the accelerated BEM is comparable to that of the conventional BEM. Both sets of results are very close to the analytical solutions. The fluctuation observed in some errors corresponding to a few fine meshes is likely caused by the round-off error. Fig. 2 displays the mean radial displacement as a function of the radius obtained by the present BEM and the analytical solutions. From this figure, it is clear that the numerical results agree very well with the analytical solutions except at some points close to the inner and outer surfaces when the discretization is coarse. The cause of these large errors is the nearly singular integrals which occur when the element size is much large compared to the distance between the evaluation points to the surfaces. In the current implementation, no special treatment has been done to regularize these nearly singular integrals. It is however not difficult to employ a non-linear transformation technique [11] to eliminate near singularity and produce accurate results. The efficiency of the accelerated BEM is demonstrated in Table 2 where the computational time at different discretization levels are listed. The conventional BEM with Gauss elimination fails to simulate the problems with more than 4500 panels due to the exhaustion of the physical memory.

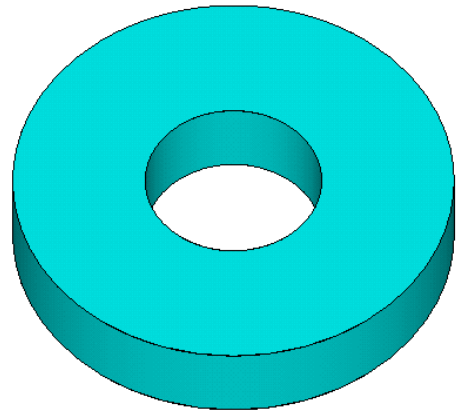

(a)

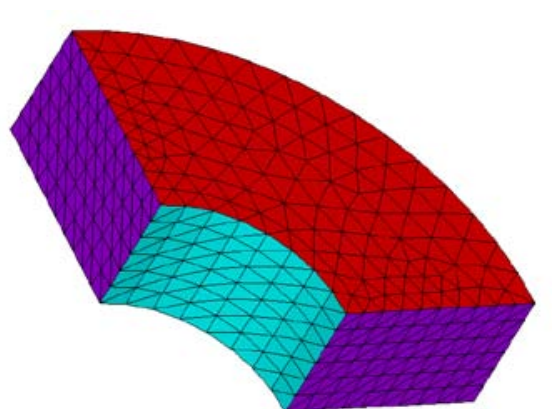

(b)

FIGURE 1. (a) Solid model of a pressurized thick cylinder, (b) BEM mesh with 950 panels.

TABLE 1. Relative errors at different discretization levels

\begin{tabular}{lcccccccc}
\hline Panels & \multicolumn{2}{c}{$u_{r}$ at $r=10 \mu m$, analytical: 10.363637e-5 } & \multicolumn{2}{c}{$u_{r}$ at $r=25 \mu m$, analytical: 5.772728e-5 } \\
\cline { 2 - 8 } & \multicolumn{2}{c}{ BEM with PFFT } & \multicolumn{2}{c}{ Conventional BEM } & \multicolumn{2}{c}{ BEM with PFFT } & \multicolumn{2}{c}{ Conventional BEM } \\
\cline { 2 - 8 } & value & error & value & error & value & error & value & error \\
\hline 102 & $10.81 \mathrm{E}-5$ & $4.31 \%$ & $10.83 \mathrm{E}-5$ & $4.50 \%$ & $5.851 \mathrm{E}-5$ & $1.36 \%$ & $5.866 \mathrm{E}-5$ & $1.62 \%$
\end{tabular}




\begin{tabular}{lcccccccc}
252 & $10.49 \mathrm{E}-5$ & $1.20 \%$ & $10.50 \mathrm{E}-5$ & $1.30 \%$ & $5.755 \mathrm{E}-5$ & $0.31 \%$ & $5.775 \mathrm{E}-5$ & $0.04 \%$ \\
950 & $10.45 \mathrm{E}-5$ & $0.83 \%$ & $10.48 \mathrm{E}-5$ & $1.12 \%$ & $5.761 \mathrm{E}-5$ & $0.20 \%$ & $5.783 \mathrm{E}-5$ & $0.18 \%$ \\
4500 & $10.40 \mathrm{E}-5$ & $0.35 \%$ & --- & & $5.770 \mathrm{E}-5$ & $0.05 \%$ & --- & \\
18000 & $10.38 \mathrm{E}-5$ & $0.12 \%$ & --- & & $5.756 \mathrm{E}-5$ & $0.29 \%$ & --- & \\
\hline
\end{tabular}

TABLE 2. Computational time at different discretization levels (computer: Dell Latitude D630 CPU 2.0GHz,

\begin{tabular}{lcc} 
& \multicolumn{2}{c}{ RAM 2.0GB) } \\
\cline { 2 - 3 } Panels & \multicolumn{2}{c}{ Time (seconds) } \\
\hline 102 & BEM with PFFT & Conventional BEM \\
252 & 2 & 1 \\
950 & 10 & 11 \\
4500 & 28 & 264 \\
18000 & 128 & --- \\
\hline
\end{tabular}

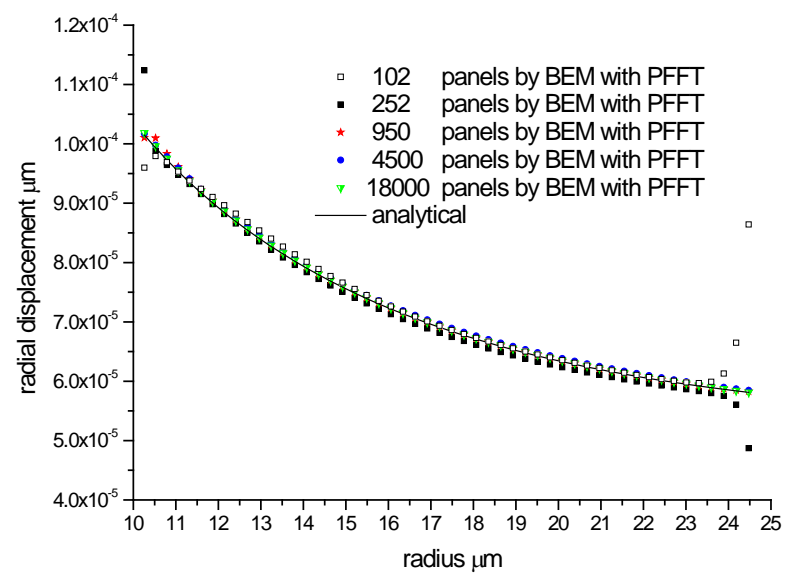

FIGURE 2. Radial displacement of the pressurized thick cylinder.

Case 2: The second testing example is a unit cube with 40 identical spherical cavities as shown in Fig. 3 . The radius of the spheres is set to be 0.106 . The material properties are the same as those of the cylinder in case 1 . The total number of boundary elements is 48,330 as shown in Fig. 3(a). For this model, the accelerated BEM runs about 16 minutes on a PC Dell OPTIPLEX 755 with a convergence tolerance set to be $10^{-8}$. For comparison, FEM simulation by ANSYS is also carried out. Unstructural mesh with a total of 63,574 SOLID92 elements is employed as shown in Fig. 3(b). The meshing advantage of the BEM is clearly demonstrated.

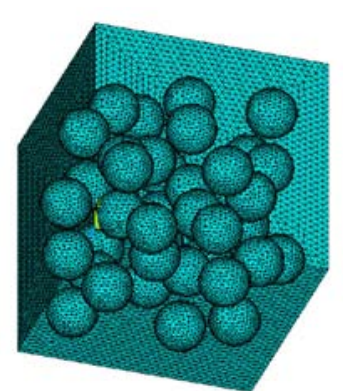

(a)

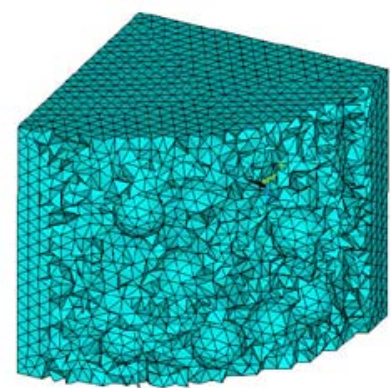

(b)

FIGURE 3. (a) BEM mesh, (b) part of FEM mesh. The boundary conditions for the cube are: bottom surface: fixed; top surface: uniform normal traction; lateral surfaces: traction free.

The computed vertical displacements on the top surface $z=1$ along the line $x=0.5$ are plotted in Fig. 4. Overall the correspondence between the two sets of results is pretty good given the fact that only 48,330 constant elements 
are employed in the BEM simulation while 63,574 quadratic elements are employed in ANSYS calculation. The largest relative error is about $3.62 \%$ at $y=0.956$.

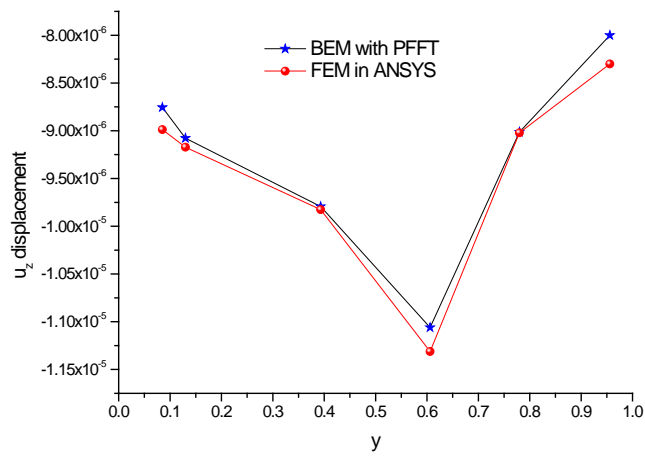

FIGURE 4. Displacement $u_{z}$ on the surface $z=1$ along the line $x=0.5$ obtained from the FEM and the accelerated BEM calculations.

\section{Preliminary Study of Porous Solids with Spherical Cavities}

The Young's modulus and the Poisson ratio of a porous solid with spherical cavities are investigated using the developed accelerated BEM code. Particular emphasize is placed on the effect of cavitie distribution and size on the overall material properties. A unit cube with various spherical cavities is constructed as the representative model for the porous solid of interest. Different distributions of cavities at a given porosity are generated using the RSA algorithm. Figure 5 shows two models with the same number and the same size of cavities but with different distributions. The porosity, characterized by volume fraction $\zeta$, in these two models is 0.2 . In addition, models with different number $(N)$, and thus the different size, of cavities at a given porosity are also generated to study the size effect. Figures 6 and 7 plot the relative Young's modulus and Poisson ratios computed from various models.

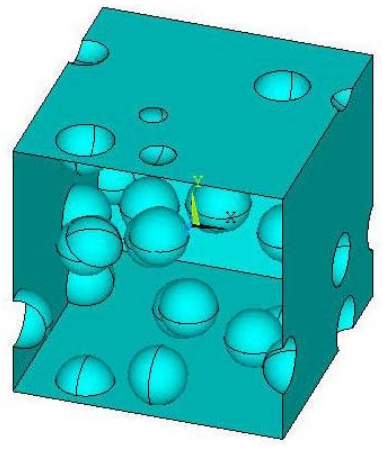

(a)

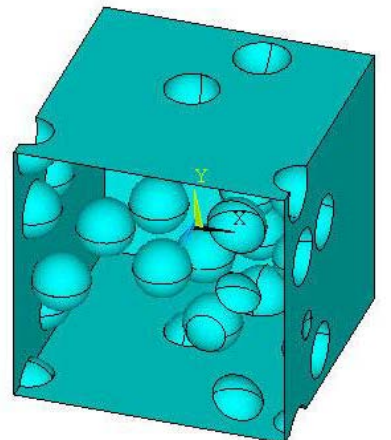

(b)

FIGURE 5. Geometric models of a unit cube with spherical cavities.
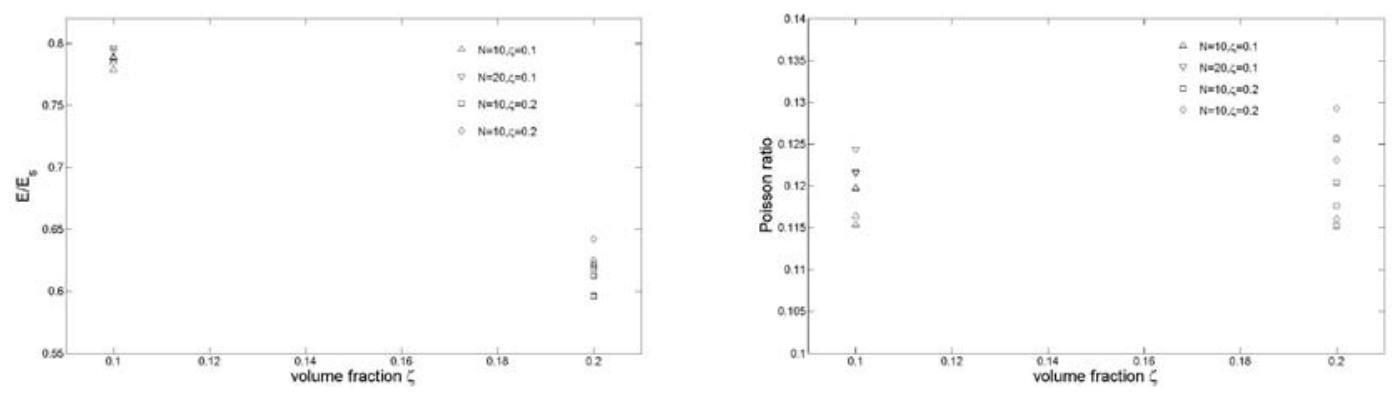

FIGURE 6. Relative Young's modulus and Poisson ratio as functions of volume fraction. 
Based on the data presented in Figure 6, the following conclusions might be drawn. First, as expected, the Young's modulus decreases as the volume fraction increases. Second, the distribution of cavities has a negligible influence on the Young's modulus when the porosity is small, indicating the Young's modulus of the porous solid is still dominated by that of the solid phase. As the porosity increases, the influence of cavity distribution becomes stronger. Last, the size of the cavities, characterized by the ratio of the radius of spheres and the unit length of the cube, also has some influence on the Young's modulus in addition to the volume fraction. This is reflected by the variation it produces in the Young's modulus for a given volume fraction. This variation is larger than the one caused by distribution. The Poisson ratio, on the other hand, seems to be largely influenced by the distribution. No qualitative conclusions can be drawn at this stage.

\section{CONCLUSIONS}

A 3-D precorrected FFT accelerated BEM approach is proposed and developed for the linear elastic analysis of porous solids. The developed BEM code is validated using analytical examples and finite element calculations based on ANSYS. The accuracy and the efficiency of the BEM approach are demonstrated on a linear elastic analysis of a porous solid with 40 spherical cavities. A preliminary study on the Young's modulus and the Poisson ratio of a porous solid with spherical cavities is conducted. It has been found that in addition to the volume fraction, both the distribution and the size of pores seem to have some influence on the Young's modulus, particularly when the porosity is large. Further investigation is required in order to quantitatively study these effects.

\section{REFERENCES}

1. A. P. Robertsa; E. J. Garboczi, "Elastic properties of model random three-dimensional open-cell solids," Journal of Mechanics and Physics of Solids, 50, pp33-55, 2002.

2. O. Caty, E. Maire, S. Youssef, R. Bouchet, "Modeling the properties of closed-cell cellular materials from tomography images using finite shell elements”, Acta Materialia, 56, pp5524-5534, $2008 .$.

3. P. K. Banerjee, The boundary element method in engineering, McGraw-Hill Book Company, pp94-130, 1994.

4. L. Greengard, V. Rokhlin, "A new version of the fast multipole method for the Laplace equation in three dimensions”, Acta Numerica, pp. 229-269, 1997.

5. J. R. Phillips, J. K. White, “A precorrected-FFT method for electrostatic analysis of complicated 3-D structures”, IEEE Transactions on Computer-Aided Design of Integrated Circuits and Systems, 16(10), pp.1059-1072, 1997.

6. W. Ye, X. Wang, W. Hemmert, D. Freeman and J. White, “Air damping in lateral oscillating micro resonators: a numerical and experimental study”, ASME/IEEE Journal of Microelectromechanical Systems, 12(5), pp. 557-566, 2003.

7. Y. J. Liu, N. Nishimura, Y. Otani, T. Takahashi, X. L. Chen, and H. Munakata, "A fast boundary element method for the analysis of fiber-reinforced composites based on a rigid-inclusion model”, Journal of Applied Mechanics, 72, pp115-128, 2005.

8. J. Segurado, J. Llorca, "A numerical approximation to the elastic properties of sphere-reinforced composites", Journal of Mechanics and Physics of Solids, 50, pp2107-2121, 2002.

9. Y. Saad and M. Schultz, GMRES: “A generalized minimal residual algorithm for solving symmetric linear systems”, SIAM Journal on Scientific and Statistical Computing, 7, pp. 856-869, 1986.

10. T. A. Cruse, "Numerical solutions in three-dimensional elastostatics”, Int J Solids Struct, 5, 1259-1274, 1969.

11. W. Ye, “A new transformation technique for evaluating nearly singular integrals”, Computational Mechanics, 42(3), pp. 457466, 2008.

\section{ACKNOWLEDGMENTS}

The contents presented in this paper were supported by Hong Kong Research Grants Council under Competitive Earmarked Research Grant 621607. Their financial support is gratefully acknowledged. 